\title{
Inflammatory prognostic scoring systems are risk factors for surgical site infection following wide local excision of soft tissue sarcoma
}

\author{
Omer M. Farhan-Alanie ${ }^{1} \oplus$ - Taegyeong Tina $\mathrm{Ha}^{1} \cdot$ James Doonan $^{1} \cdot$ Ashish Mahendra $^{1} \cdot$ Sanjay Gupta ${ }^{1}$
}

Received: 13 September 2021 / Accepted: 30 September 2021 / Published online: 9 October 2021

(c) The Author(s) 2021

\begin{abstract}
Introduction Limb-sparing surgery with negative margins is possible in most soft tissue sarcoma (STS) resections and focuses on maximising function and minimising morbidity. Various risk factors for surgical site infections (SSIs) have been reported in the literature specific to sarcoma surgery. The aim of this study is to determine whether systemic inflammatory response prognostic scoring systems can predict post-operative SSI in patients undergoing potentially curative resection of STS.

Methods Patients who had a planned curative resection of a primary STS at a single centre between January 2010 and December 2019 with a minimum follow-up of 6 months were included. Data were extracted on patient and tumour characteristics, and pre-operative blood results were used to calculate inflammatory prognostic scores based on published thresholds and correlated with risk of developing SSI or debridement procedures.

Results A total of 187 cases were included. There were 60 SSIs. On univariate analysis, there was a statistically significant increased risk of SSI in patients who are diabetic, increasing specimen diameter, American Society of Anaesthesiology (ASA) grade 3, use of endoprosthetic replacement, blood loss greater than $1 \mathrm{~L}$, and junctional tumour location. Modified Glasgow prognostic score, C-reactive protein/albumin ratio and neutrophil-platelet score (NPS) were statistically associated with the risk of SSI. On multivariate analysis, ASA grade 3, junctional tumour location and NPS were independently associated with the risk of developing a SSI.

Conclusion This study supports the routine use of simple inflammation-based prognostic scores in identifying patients at increased risk of developing infectious complications in patients undergoing potentially curative resection of STS.
\end{abstract}

Keywords Soft tissue sarcoma $\cdot$ Infection $\cdot$ Inflammatory prognostic score $\cdot$ Modified Glasgow prognostic score

\section{Introduction}

The principles of management of soft tissue sarcoma (STS) have evolved considerably in recent years. Limb-sparing surgery with negative margins is possible in the majority of resections and focuses on maximising function and minimising morbidity $[1,2]$. Amputation is only required in approximately $10 \%$ of cases, where the involvement of critical anatomic structures prevents a curative wide local surgical resection [3]. However, limb-sparing resections often involve large surgical fields, and surgical site infection

Omer M. Farhan-Alanie

omeralanie@doctors.org.uk

1 Department of Musculoskeletal Oncology Surgery, Glasgow Royal Infirmary, 84 Castle St, Glasgow G4 0SF, United Kingdom
(SSI) remains an important source of post-operative morbidity [4]. Wound complications including dehiscence, cellulitis, abscess, seromas, haematomas and wound necrosis have been reported to occur in $16-56 \%$ cases in the published literature $[4,5]$.

Various risk factors for surgical site infections have been reported in the literature specific to sarcoma surgery, and these can be sub-classified into surgical factors, host factors and adjunctive treatment factors [5-7]. Large tumour resections, particularly involving the adductor compartment of the thigh, use of adjuvant radiotherapy, and a variety of patient-specific factors including smoking, diabetes and obesity, have all been reported to independently affect the risk of SSIs [8]. Identification and knowledge of such risk factors is important as it allows appropriate patient counselling and can guide future research in reducing these risks. Recent proposals have focussed on these high risk wounds 
and look to utilise existing technology such as negative pressure wound therapy to mitigate these risk factors [9]. There is also evidence to support the immediate use of free-flap reconstruction, particularly in patients who receive neoadjuvant radiotherapy, in an effort to substitute irradiated soft tissue for healthy soft tissue from the donor site, and optimising the vascularisation of the resection site [10].

There has been increasing evidence over the last two decades that the host response to malignancy plays a key role in the prognosis and outcomes of treatment. A systemic inflammatory response is a proposed unifying model for this host interaction with the tumour, with various scoring systems proposed to quantify this [11-14]. These scoring systems rely on routine haematological and biochemical laboratory parameters to stratify this response, and they have been shown in numerous studies to correlate with survival in a variety of malignancies $[13,15]$. More recent work has focussed on their use in predicting post-operative morbidity, particularly in potentially curative carcinoma resections [16].

However, only limited data are available on the applicability of systemic inflammatory response prognostic scores in the management of soft tissue sarcomas, which represent a different cellular lineage to carcinomas [17]. Emerging evidence supports a correlation between the modified Glasgow prognostic score (mGPS) and survival in soft tissue sarcoma, but no studies have investigated their relationship with postoperative SSIs in soft tissue sarcoma. Therefore, the aim of this study is to determine whether systemic inflammatory response prognostic scoring systems can independently predict post-operative surgical site infection in patients undergoing potentially curative resection of soft tissue sarcoma.

\section{Methods}

All patients who had a planned curative resection of a primary STS in the West of Scotland between the calendar years January 2010 and December 2019 with a minimum follow-up of 6 months were entered into the study. These patients were identified from a prospectively maintained database. Review of the electronic case notes was performed for 213 patients who met the inclusion criteria. Any patient with metastatic disease at presentation, a low grade tumour subtype (atypical lipomatous tumour/dermatofibrosarcoma protuberans) or incomplete data were excluded to avoid selection and transfer bias. As a result of access to a national electronic case note files, no patients were lost to follow-up.

All patients were treated surgically under the supervision of the two senior authors (AM and SG). Data were extracted on sex, age, height, weight, use of adjuvant therapy, comorbidities, American Society of Anaesthesiology (ASA) grade, intra-operative blood loss, tumour size, tumour grade, specimen size, location of tumour and need for immediate soft tissue reconstruction. We grouped tumour location into anatomic location but described a subset which we defined as junctional, which occur in the axilla, groin, popliteal fossa and distal medial thigh. All patients received a low-pressure vacuum drain in the immediate post-operative period, which was left in situ until drainage was less than $50 \mathrm{ml}$ over a 24-h period. Peri-operative antibiotic prophylaxis was routinely continued until drain removal. Thrombo-embolic prophylaxis consisted of low-molecular weight heparin, which was maintained as inpatient therapy for all patients, and for 4 weeks post-operatively in lower limb sarcoma surgery.

Pre-operative blood results including C-reactive protein, albumin, white cell count, neutrophil, lymphocyte and platelet count were collected. These data were used to calculate established inflammatory scoring systems based on validated thresholds as demonstrated in Table 1 [13].

Data were collected on post-operative surgical site infections (SSIs). Patients were routinely followed up at 2 weeks post-operatively at the sarcoma clinic, then at regular intervals thereafter until the wound is satisfactory. If patients underwent soft tissue reconstruction, then the immediate post-operative follow-up was by the onco-plastic team weekly for the first 4 weeks. If post-operative radiotherapy was recommended, this was instituted once the wound was deemed satisfactory by the surgical team, and standard treatment was to commence this at 6 weeks post-operatively. We defined a post-operative SSI as a surgical site requiring treatment with antibiotic therapy or an infective complication that required surgical intervention such as debridement and washout of the surgical site. No ethical approval was required for this study.

\section{Statistics}

Variables were groups by standard binary or categorical thresholds. Univariate survival analysis was performed using a Cox proportional hazards model taking into account time to surgical site infection. Kaplan-Meir analysis using logrank test was used to graphically demonstrate significance. A $p$-value of $<0.05$ was considered statistically significant. Multivariate analysis was performed using a Cox proportional hazards model with a stepwise backward procedure to derive a final model of the variables that had a significant independent relationship with post-operative surgical site complications.

Inter-relationships between variables were assessed using contingency table analysis with the Chi square test for trend as appropriate. For variables with few observations, Fisher exact test was used. Analysis was performed using SPSS 
Table 1 Systemic inflammationbased prognostic ratios and scores

\begin{tabular}{|c|c|}
\hline Ratio/score & Ratio/score \\
\hline \multicolumn{2}{|l|}{$N L R$} \\
\hline Neutrophil count/lymphocyte count & $\leq 3$ \\
\hline Neutrophil count/lymphocyte count & $3-5$ \\
\hline Neutrophil count/lymphocyte count & $>5$ \\
\hline \multicolumn{2}{|l|}{$N L S$} \\
\hline Neutrophil count $\leq 7.5 \times 10^{9} / 1$ and lymphocyte count $\geq 1.5 \times 10^{9} / 1$ & 0 \\
\hline Neutrophil count $>7.5 \times 10^{9} / 1$ and lymphocyte count $\geq 1.5 \times 10^{9} / 1$ & 1 \\
\hline Neutrophil count $\leq 7.5 \times 10^{9} / 1$ and lymphocyte count $<1.5 \times 10^{9} / 1$ & 1 \\
\hline Neutrophil count $>7.5 \times 10^{9} / 1$ and lymphocyte count $<1.5 \times 10^{9} / 1$ & 2 \\
\hline \multicolumn{2}{|l|}{$P L R$} \\
\hline Platelet count/lymphocyte count & $\leq 150$ \\
\hline Platelet count/lymphocyte count & $>150$ \\
\hline \multicolumn{2}{|l|}{$P L S$} \\
\hline Platelet count $\leq 400 \times 10^{9} / 1$ and lymphocyte count $\geq 1.5 \times 10^{9} / 1$ & 0 \\
\hline Platelet count $>400 \times 10^{9} / 1$ and lymphocyte count $\geq 1.5 \times 10^{9} / 1$ & 1 \\
\hline Platelet count $\leq 400 \times 10^{9} / 1$ and lymphocyte count $<1.5 \times 10^{9} / 1$ & 1 \\
\hline Platelet count $>400 \times 10^{9} / 1$ and lymphocyte count $<1.5 \times 10^{9} / 1$ & 2 \\
\hline \multicolumn{2}{|l|}{$L M R$} \\
\hline Lymphocyte count/monocyte count & $\geq 2.40$ \\
\hline Lymphocyte count/monocyte count & $<2.40$ \\
\hline \multicolumn{2}{|l|}{$L M S$} \\
\hline Lymphocyte count $\geq 1.5 \times 10^{9} / 1$ and monocyte count $\leq 0.8 \times 10^{9} / 1$ & 0 \\
\hline Lymphocyte count $\geq 1.5 \times 10^{9} / 1$ and monocyte count $\leq 0.8 \times 10^{9} / 1$ & 1 \\
\hline Lymphocyte count $<1.5 \times 10^{9} / 1$ and monocyte count $>0.8 \times 10^{9} / 1$ & 1 \\
\hline Lymphocyte count $<1.5 \times 10^{9} / 1$ and monocyte count $>0.8 \times 10^{9} / 1$ & 2 \\
\hline \multicolumn{2}{|l|}{$N P S$} \\
\hline Neutrophil count $\leq 7.5 \times 10^{9} / 1$ and platelet count $<400 \times 10^{9} / 1$ & 0 \\
\hline Neutrophil count $>7.5 \times 10^{9} / 1$ and platelet count $<400 \times 10^{9} / 1$ & 1 \\
\hline Neutrophil count $\leq 7.5 \times 10^{9} / 1$ and platelet count $>400 \times 10^{9} / 1$ & 1 \\
\hline Neutrophil count $>7.5 \times 10^{9} / 1$ and platelet count $>400 \times 10^{9} / 1$ & 2 \\
\hline \multicolumn{2}{|l|}{$C A R$} \\
\hline C-reactive protein/albumin & $\leq 0.22$ \\
\hline C-reactive protein/albumin & $>0.22$ \\
\hline \multicolumn{2}{|l|}{$m G P S$} \\
\hline C-reactive protein $\leq 10 \mathrm{mg} / \mathrm{l}$ and albumin $\geq 35 \mathrm{~g} / \mathrm{l}$ & 0 \\
\hline C-reactive protein $>10 \mathrm{mg} / \mathrm{l}$ and albumin $\geq 35 \mathrm{~g} / \mathrm{l}$ & 1 \\
\hline C-reactive protein $>10 \mathrm{mg} / \mathrm{l}$ and albumin $<35 \mathrm{~g} / \mathrm{l}$ & 2 \\
\hline
\end{tabular}

$N L R$ Neutrophil-lymphocyte ratio; $N L S$ neutrophil-lymphocyte score; $P L R$ platelet-lymphocyte ratio; $P L S$ platelet-lymphocyte score; $L M R$ lymphocyte-monocyte ratio; $L M S$ lymphocyte-monocyte score; NPS neutrophil-platelet score; $C A R$ C-reactive protein-albumin ratio; $m G P S$ modified Glasgow prognostic score software (version 26.0.0. SPSS Inc, Chicago, IL, USA) or GraphPad Prism (version 6, San Diego, CA, USA).

\section{Results}

Between January 2010 and December 2019, there were 187 cases eligible for analysis. A summary of the clinicopathological characteristics of the patients is presented in
Table 2. There were 60 surgical site infections; 21 of these were treated with antibiotic therapy, and 39 patients required an additional surgical procedure. Median time to diagnosis of any surgical site infection was 22 days (interquartile range 14-40 days), and median time to surgical intervention was 21 days (interquartile range 16-34 days). There were 21 distinct subtypes of soft tissue sarcomas within our group, and these are summarised in Table 3. 
Table 2 Variable distribution and univariate Cox regression analysis

\begin{tabular}{|c|c|c|c|c|c|}
\hline Variable & Total & No complication & Complication & $p$-value & Hazard ratio $(95 \% \mathrm{CI})$ \\
\hline Number of patients & 187 & 127 & 60 & - & \\
\hline Sex & & & & 0.938 & \\
\hline Male & 90 & 61 & 29 & & \\
\hline Female & 97 & 66 & 31 & & \\
\hline Mean age in years (Range) & $59.0(19-93)$ & $59(20-91)$ & $59(19-93)$ & 0.960 & \\
\hline Mean BMI (range) & $28.3(17.7-68.5)$ & $27.9(17.7-68.5)$ & $29.1(19.4-55.5)$ & 0.219 & \\
\hline Trojani tumour grade & & & & 0.175 & \\
\hline Grade 1 & 16 & 13 & 3 & & \\
\hline Grade 2 & 49 & 35 & 14 & & \\
\hline Grade 3 & 122 & 79 & 43 & & \\
\hline Mean tumour diameter in $\mathrm{cm}$ (range) & $9.42(0.5-31.0)$ & $9.0(0.5-31.0)$ & $10.3(1.3-27.5)$ & 0.206 & \\
\hline Mean specimen diameter in $\mathrm{cm}$ (range) & $16.1(2.0-39.0)$ & $14.9(2.0-39.0)$ & $18.7(3.0-34.0)$ & 0.003 & $1.06(1.02-1.09)$ \\
\hline Tumour site & & & & & $4.69(1.11-19.76)$ \\
\hline Trunk & 15 & 13 & 2 & - & \\
\hline Upper limb & 35 & 29 & 6 & 0.73 & \\
\hline Lower limb & 86 & 60 & 26 & 0.218 & \\
\hline Junctional & 51 & 25 & 26 & 0.035 & \\
\hline \multicolumn{6}{|l|}{ Adjuvant radiotherapy } \\
\hline None & 57 & 42 & 15 & - & \\
\hline Adjuvant & 98 & 66 & 32 & 0.615 & \\
\hline Neoadjuvant & 32 & 19 & 13 & 0.217 & \\
\hline Wound closure & & & & 0.486 & \\
\hline Primary & 95 & 67 & 28 & & \\
\hline Soft tissue reconstruction & 92 & 60 & 32 & & \\
\hline \multicolumn{6}{|l|}{ ASA grade } \\
\hline II & 143 & 105 & 38 & - & $2.42(1.42-4.12)$ \\
\hline III & 39 & 18 & 21 & 0.001 & \\
\hline IV & 5 & 4 & 1 & 0.812 & \\
\hline Diabetes mellitus & & & & 0.045 & $1.95(1.01-3.76)$ \\
\hline Yes & 165 & 116 & 11 & & \\
\hline No & 22 & 11 & 49 & & \\
\hline Hypertension & & & & 0.147 & \\
\hline Yes & 118 & 15 & 8 & & \\
\hline No & 68 & 112 & 51 & & \\
\hline Hypercholesterolaemia & 163 & 15 & 8 & 0.819 & \\
\hline Yes & 23 & 112 & 51 & & \\
\hline \multicolumn{6}{|l|}{ No } \\
\hline Smoker & & & & 0.744 & \\
\hline Active & 30 & 20 & 10 & & \\
\hline Non-active & 157 & 107 & 50 & & \\
\hline Surgery type & & & & 0.646 & \\
\hline Ablative & 26 & 19 & 7 & & \\
\hline Limb sparing & 161 & 108 & 53 & & \\
\hline Bone reconstruction & & & & 0.028 & $2.43(1.10-5.35)$ \\
\hline Nil & 177 & 124 & 53 & & \\
\hline Endoprosthesis & 10 & 3 & 7 & & \\
\hline Blood loss & & & & 0.022 & $2.22(1.12-4.38)$ \\
\hline$<1$ Litre & 170 & 120 & 50 & & \\
\hline$>1$ Litre & 17 & 7 & 10 & & \\
\hline
\end{tabular}


Table 2 (continued)

\begin{tabular}{|c|c|c|c|c|c|}
\hline Variable & Total & No complication & Complication & $p$-value & Hazard ratio $(95 \% \mathrm{CI})$ \\
\hline mGPS & & & & 0.019 & $1.41(1.06-1.88)$ \\
\hline 0 & 108 & 81 & 27 & & \\
\hline 1 & 38 & 24 & 14 & & \\
\hline 2 & 41 & 22 & 19 & & \\
\hline CAR & & & & 0.04 & $1.67(1.004-2.79)$ \\
\hline 0 & 100 & 74 & 26 & 8 & \\
\hline 1 & 84 & 50 & 34 & & \\
\hline NLR & & & & 0.182 & \\
\hline 0 & 89 & 65 & 24 & & \\
\hline 1 & 58 & 37 & 12 & & \\
\hline 2 & 38 & 23 & 15 & & \\
\hline NLS & & & & 0.230 & \\
\hline 0 & 99 & 70 & 29 & & \\
\hline 1 & 78 & 51 & 27 & & \\
\hline 2 & 8 & 4 & 4 & & \\
\hline PLR & & & & 0.103 & \\
\hline 0 & 60 & 46 & 14 & & \\
\hline 1 & 125 & 79 & 46 & & \\
\hline PLS & & & & 0.131 & \\
\hline 0 & 94 & 67 & 27 & & \\
\hline 1 & 84 & 56 & 28 & & \\
\hline 2 & 7 & 2 & 5 & & \\
\hline NPS & & & & 0.004 & $1.73(1.19-2.52)$ \\
\hline 0 & 148 & 107 & 41 & & \\
\hline 1 & 28 & 15 & 13 & & \\
\hline 2 & 9 & 3 & 6 & & \\
\hline
\end{tabular}

$\mathrm{BMI}=$ body mass index, $\mathrm{CI}=$ confidence interval

Bold represents statistically significant finding

Univariate analysis determined that there was a statistically significant increased risk of SSI in patients who are diabetic (50\% versus $29.7 \%, p=0.045)$ and patients with an ASA grade $3(53.8 \%$ versus $26.6 \%$ ASA 2 patients, $p=0.001)$. There was no significant association between other patient related risk factors such as sex, age, smoking status, Body Mass Index (BMI), hypertension or hypercholesterolaemia.

With regard to pathological characteristics, the mean tumour diameter was $9.42 \mathrm{~cm}$ and the mean specimen diameter was $16.1 \mathrm{~cm}$. The majority of tumours were Trojani grade $3(65.2 \%)$. There was a statistically significant $6 \%$ increased risk of complications per centimetre increase in the maximum measured specimen diameter $(p=0.003)$. There was no association between risk of complication and maximum measured tumour diameter or Trojani tumour grade.

Surgical factors associated with increased risk of postoperative SSI include intra-operative blood loss greater than $1 \mathrm{~L}(58.8 \%$ versus $29.4 \%, p=0.022)$, junctional location of tumour (51\% versus $17.1 \%$ in upper limb, $p=0.035$ ) and implantation of an endoprosthesis (70\% versus $29.9 \%$, $p=0.028$ ). There was no statistically significant association with need for primary amputation, neoadjuvant or adjuvant radiotherapy or type of wound closure.

We examined the association of SSI with various previously described systemic inflammatory scoring systems. There was a statistically significant association between the modified Glasgow prognostic scoring system (mGPS), C-reactive protein-to-albumin ratio (CAR), neutrophil-platelet score (NPS) and risk of developing a surgical site infection (all $p<0.05$ ). There was no statistically significant association on univariate analysis with the neutrophil-lymphocyte ratio (NLR), neutrophil-lymphocyte score (NLS), platelet-lymphocyte ratio (NLR) and platelet-lymphocyte score (PLS) and the risk of developing post-operative SSI.

On multivariate analysis, we identified three independent risk factors for developing a post-operative SSI (Table 4). 
Table 3 Tumour histology types included in study

\begin{tabular}{ll}
\hline Tumour type & $n$ \\
\hline Undifferentiated sarcoma NOS & 34 \\
Undifferentiated pleomorphic sarcoma & 25 \\
Myxoid liposarcoma & 24 \\
Spindle cell sarcoma & 24 \\
Myxofibrosarcoma & 18 \\
Leiomyosarcoma & 17 \\
Synovial sarcoma & 10 \\
Liposarcoma & 7 \\
Rhabdomyosarcoma & 5 \\
Malignant peripheral nerve sheath & 5 \\
Extraskeletal osteosarcoma & 3 \\
Fibromyxoid sarcoma & 3 \\
Angiosarcoma & 3 \\
Myofibroblastic sarcoma & 2 \\
Pleomorphic hyalinising angiectatic & 1 \\
Epithelioid sarcoma & 1 \\
Small cell neuroendocrine & 1 \\
Clear cell sarcoma & 1 \\
Fibromyxoid sarcoma & 1 \\
Myxoinflammatory fibroblastic sarcoma & 1 \\
Round cell sarcoma & 1 \\
\hline
\end{tabular}

NOS: not otherwise specified

Table 4 Statistical association between variables included in study and systemic inflammatory scoring systems significant on univariate analysis

\begin{tabular}{lrrr}
\hline Variable & \multicolumn{1}{l}{ NPS } & \multicolumn{1}{l}{ CAR } & \multicolumn{1}{c}{ mGPS } \\
\hline Sex & 0.333 & 0.685 & 0.584 \\
Age & $\mathbf{0 . 0 3 4}$ & $<\mathbf{0 . 0 0 1}$ & $\mathbf{0 . 0 0 2}$ \\
BMI & 0.710 & 0.083 & 0.106 \\
Trojani tumour grade & $\mathbf{0 . 0 1 6}$ & $<\mathbf{0 . 0 0 1}$ & $<\mathbf{0 . 0 0 1}$ \\
Tumour diameter & $\mathbf{0 . 0 0 1}$ & $\mathbf{< 0 . 0 0 1}$ & $<\mathbf{0 . 0 0 1}$ \\
Specimen diameter & $\mathbf{0 . 0 0 4}$ & $<\mathbf{0 . 0 0 1}$ & $<\mathbf{0 . 0 0 1}$ \\
Tumour site & 0.199 & 0.313 & 0.245 \\
Adjuvant radiotherapy & 0.103 & $\mathbf{0 . 0 1 4}$ & 0.054 \\
Wound closure & 0.478 & $\mathbf{0 . 0 0 8}$ & 0.054 \\
ASA grade & $\mathbf{0 . 0 4 1}$ & 0.399 & $\mathbf{0 . 0 0 2}$ \\
Diabetes mellitus & 0.901 & 0.663 & 0.818 \\
Hypertension & 0.572 & 0.392 & 0.179 \\
Hypercholesterolaemia & 0.079 & 0.519 & 0.788 \\
Smoker & 0.916 & 0.060 & 0.352 \\
Surgery type & 0.215 & $\mathbf{0 . 0 2 9}$ & $\mathbf{0 . 0 2 7}$ \\
Bone reconstruction & 0.161 & 0.516 & 0.393 \\
Blood loss & $\mathbf{0 . 0 0 1}$ & $\mathbf{0 . 0 4 0}$ & $<\mathbf{0 . 0 0 1}$ \\
\hline
\end{tabular}

Bold represents statistically significant finding
The NPS was significantly and independently associated with an increased risk of SSI, with a hazard ratio of 1.64 (95\% CI 1.11-2.40) per level increase in value $(p=0.012)$. Additionally, ASA grade 3 was associated with a hazard ratio of 2.24 (1.30-3.85) when compared to ASA grade $2(p=0.004)$, and a tumour located in a junctional area was associated with a hazard ratio of 3.74 (1.53-9.12) for developing a SSI $(p=0.004)$. Figure 1 demonstrates the Kaplan-Meir survival curves for these factors.

We examined associations between the variables collected and the three systemic inflammatory scoring systems that were statistically significant on univariate analysis, namely mGPS, CAR and NPS (Table 5). There was a statistically significant association of all three scoring systems with increasing age, higher-grade tumour, increased tumour diameter size and specimen size, and increased intra-operative blood loss. NPS and mGPS were both statistically significantly associated with increased ASA score. Patients with an elevated mGPS and CAR were more likely to undergo ablative surgery. Patients with an elevated CAR were statistically significantly associated with an increased rate of soft tissue reconstruction.

\section{Discussion}

This study has demonstrated that the pre-operative systemic inflammatory prognostic scores, namely the modified Glasgow prognostic score, the C-reactive protein-to-albumin ratio and the neutrophil-platelet score, were all statistically significant prognostic factors for development of SSI in patients undergoing potentially curative resection of soft tissue sarcoma, with the latter also being an independent risk factor on multivariate analysis.

Post-operative SSI is relatively common in patients undergoing resection of soft tissue sarcoma, associated with increased hospital stay, treatment costs and may result in delays to adjuvant radiotherapy. Therefore, identifying atrisk patients is key to informed consent, but can also allow targeted interventions to mitigate these risks. The results of this study suggest that patients with an elevated NPS, ASA grade 3 or tumours resected from a junctional location may benefit from such interventions. Incisional negative pressure wound therapy has been utilised in orthopaedic and nonorthopaedic surgical scenarios, both routinely and targeted in at-risk wounds and has been shown to minimise the risk of post-operative wound complications, with an ongoing randomised control trial investigating its utility in soft tissue sarcoma surgery [18-21]. In addition, it has been shown that the use of soft tissue reconstruction can minimise the risk of surgical site infections in soft tissue sarcoma surgery, particularly patients who undergo neoadjuvant radiotherapy $[22,23]$. Whilst our study found no association between the 

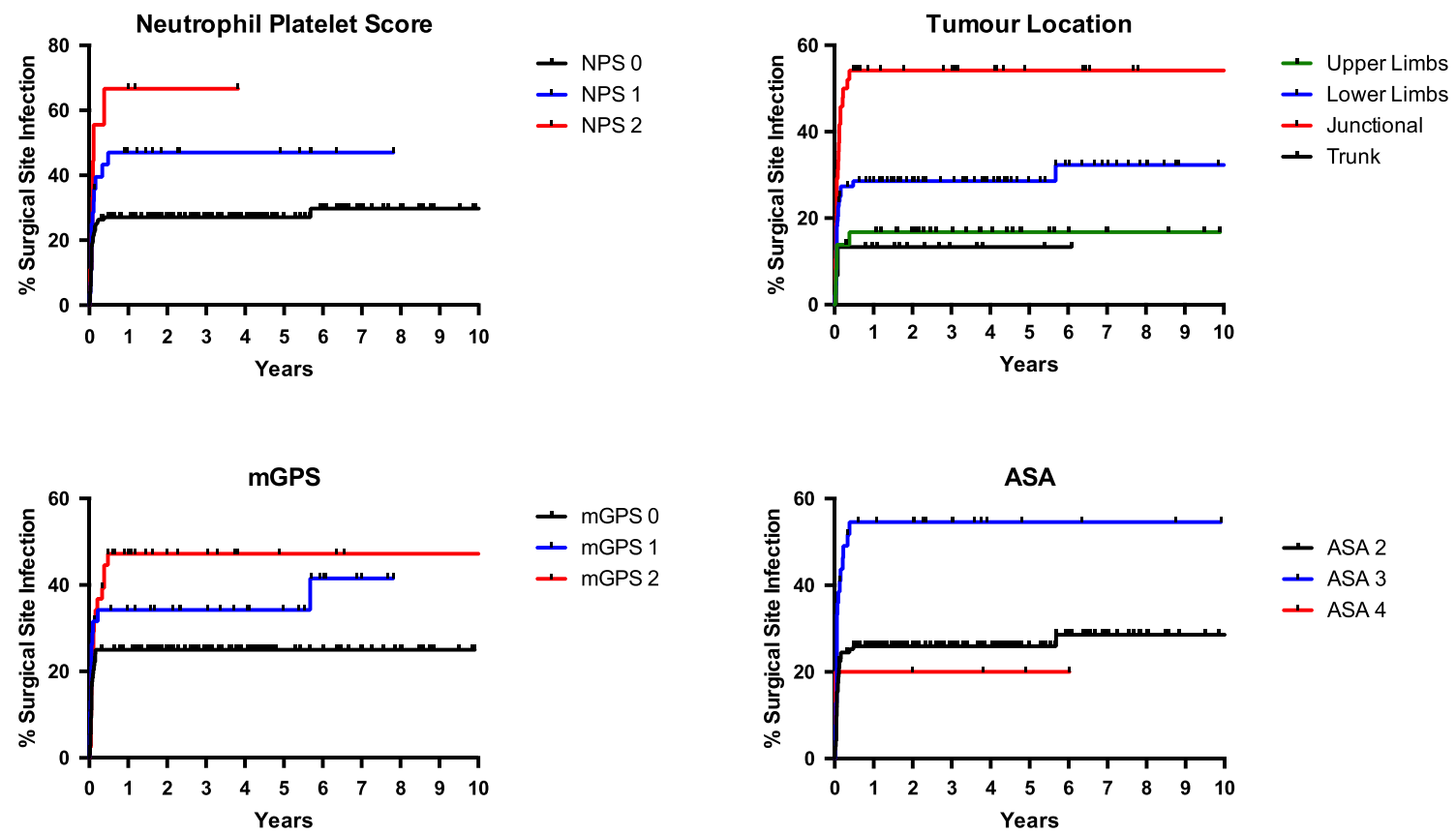

Fig. 1 Kaplan-Meir survival graph demonstrating risk of surgical site infection with a) NPS, b) mGPS, c) tumour location and d) ASA grade

Table 5 Variables determined to predict surgical site infection on multivariate analysis

\begin{tabular}{lll}
\hline Variable & $P$-value & Hazard ratio (95\% CI) \\
\hline ASA grade 3 & 0.004 & $2.24(1.29-3.88)$ \\
Neutrophil-platelet score & 0.012 & $1.64(1.11-2.40)$ \\
Junctional tumour location & 0.004 & $3.74(1.53-9.12)$ \\
\hline
\end{tabular}

use soft tissue reconstruction and risk of complications, it is likely that the retrospective nature of this study resulted in a treatment selection bias. Indeed, we noted a higher than published rate of soft tissue reconstruction in our study cohort (49.1\%), and this likely represents our evolution in practice with time to a more aggressive surgical approach using soft tissue reconstruction.

The basis of the independent relationship between an elevated systemic inflammatory response pre-operatively and post-operative infections in patients with primary operable soft tissue sarcoma is not clear. Whilst both the cell-mediated immune response and humoral immune response are associated with post-operative SSI, only the former was independently associated with complications. This is in contrast with disease-free survival in soft tissue sarcoma, which is more closely associated with the humoral immune response as measured by the mGPS [17]. It is also interesting to note that whilst the tumour size and grade were associated with an elevated mGPS, CAR and NPS, they were not associated with an increased risk of complications. We therefore hypothesise that whilst the tumour elicits a significant systemic inflammatory response in the host, it is this dysfunctional response that predisposes to the increased risk of SSI, and that these biomarkers measure the dysfunctional response rather than act as surrogates to tumour aggressiveness in the aetiology and prediction of surgical site infections.

Systemic inflammation and nutritional status are clearly inter-linked. Inflammation impairs nutritional status by reducing food intake and impairing micronutrient absorption, and malnutrition increases the risk and severity of inflammation $[24,25]$. Whilst BMI was not associated with the risk of SSI or with an increased systemic inflammatory prognostic scores, it is well recognised that absolute BMI is a poor marker of nutritional status in cancer [26, 27]. Several studies have identified a link between inflammatory prognostic scores, skeletal muscle mass and cancer cachexia, and poor nutritional status has been demonstrated to increase the risk of peri-operative complications including surgical site infections [28-30]. Further research aimed at identifying a link between inflammatory prognostic scores, cancer cachexia and surgical site infection in soft tissue sarcoma can provide easy identification of these patients and potentiate a pathway for intervening in this group to potentially reduce their risk of SSI.

It is interesting to note that the mean specimen diameter was significantly associated with risk of post-operative surgical site infections, but that tumour diameter is not. To the best of our knowledge, this is the first paper to distinguish between tumour size and specimen size in the 
same cohort. This supports the hypothesis that it is the residual dead space created by resection of larger tumours which predisposes to development of seromas and hematomas, as well as subsequent infection [4]. The significant association between increased blood loss and risk of postoperative SSI is likely driven by a similar mechanism. It has also been suggested that intra-operative blood transfusion may act synergistically with surgical stress to induce immunosuppression, and increased blood loss is likely to be associated with higher risk of intra-operative blood transfusion [31, 32].

Anatomic location of tumour is also a significant prognostic indicator of SSI [2, 8, 33]. Prior studies have focussed on anatomic divisions, but we hypothesised that grouping patients based on this feature alone can result in a dilutional effect. We have shown that these at risk areas, namely the medial thigh, popliteal fossa, groin and axilla are more appropriately grouped and are an independent risk factors for SSI. It has been postulated that certain sites are more at risk of SSI due to en bloc resection of venous and lymphatic vessels, which result in seroma and subsequent infection $[8,33]$. In addition, we propose two other features that may account for the high risk nature of these areas. There is likely a mechanical shear and tensile effect on the soft tissue in these areas, which often fall in junctional zones. In addition, these areas show a high concentration of gram negative or anaerobic flora, which may represent more opportunistic and more virulent micro-organisms [34].

We recognise that this is a heterogenous group of patients, in terms of both tumour aetiology and patient characteristics. This represents a limitation of our paper but is reflective of standard sarcoma surgeon's practice. In addition, the heterogenous nature represents a further demand for a simplified, unified method of appropriately stratifying risk of surgical site infection in this varied cohort of patients, presentations and management types.

In summary, this study supports the routine use of simple inflammation-based prognostic scores in identifying patients at increased risk of developing infectious complications in patients undergoing potentially curative resection of soft tissue sarcoma. However, it remains to be determined whether the pre-operative systemic inflammatory response may be moderated and whether such moderation may reduce post-operative infectious complications, and further prospective work is required to clarify whether interventions can be targeted in this at risk group to minimise these complications.

Funding No funding was received by the authors or institution of this study.

\section{Declarations}

Conflicts of interest On behalf of all authors, the corresponding author states that there is no conflict of interest.

Ethics approval Ethical approval was not required for this study.

Informed Consent Informed consent was not required for this study.

Open Access This article is licensed under a Creative Commons Attribution 4.0 International License, which permits use, sharing, adaptation, distribution and reproduction in any medium or format, as long as you give appropriate credit to the original author(s) and the source, provide a link to the Creative Commons licence, and indicate if changes were made. The images or other third party material in this article are included in the article's Creative Commons licence, unless indicated otherwise in a credit line to the material. If material is not included in the article's Creative Commons licence and your intended use is not permitted by statutory regulation or exceeds the permitted use, you will need to obtain permission directly from the copyright holder. To view a copy of this licence, visit http://creativecommons.org/licenses/by/4.0/.

\section{References}

1. Grimer R, Judson I, Peake D, Seddon B (2010) Guidelines for the management of soft tissue sarcomas. Sarcoma 2010:1-15

2. Korah MP, Deyrup AT, Monson DK et al (2012) Anatomic tumor location influences the success of contemporary limb-sparing surgery and radiation among adults with soft tissue sarcomas of the extremities. Int J Radiat Oncol Biol Phys 82:933-939

3. Prendergast B, Fiveash JB, Gibbs CP, Scarborough MT, Indelicato DJ (2012). Radiotherapy for soft tissue sarcoma of the proximal lower extremity. Sarcoma; 829498.

4. Geller DS, Hornicek FJ, Makin HJ, Raskin KA (2007) Soft tissue sarcoma resection volume associated with wound-healing complications. Clin Orthop Relat Res 459:182-185

5. Peat BG, Bell RS, Davis A et al (1994) Wound-healing complications after soft-tissue sarcoma surgery. Plast Reconstr Surg 93(5):980-987

6. Baldini EH, Lapidus MR, Wang Q, Manola J, Orgill DP et al (2013) Predictors for major wound complications following preoperative radiotherapy and surgery for soft tissue sarcoma of the extremity and trunk: importance of tumor proximity to the skin surface. Ann Surg Oncol 20:1494-1499

7. Hill GE, Frawley WH, Griffith KE (2003) Allogeneic blood transfusion increases the risk of postoperative bacterial infection: a meta-analysis. J Trauma 54(5):908-914

8. Moore J, Isler M, Barry J, Mottard S (2014) Major wound complication risk factors following soft tissue sarcoma resection. Eur J Surg Oncol 40(12):1671-1676

9. Kong R, Shields D, Bailey O, Gupta S, Mahendra A (2017) Negative Pressure Wound Therapy for Closed Surgical Wounds in Musculoskeletal Oncology Patients - A Case-Control Trial. Open Orthop J 11:502-507

10. Chao AH, Chang DW, Shuaib SW, Hanasono MM (2012) The effect of preoperative versus postoperative irradiation on microvascular free flap reconstruction in sarcoma patients. Plast Reconstr Surg 129(3):675-682

11. Diakos CI, Charles KA, McMillan DC, Clarke SJ (2014) Cancerrelated inflammation and treatment effectiveness. Lancet Oncol 15(11):e493-e503 
12. Roxburgh CS, McMillan DC (2010) Role of systemic inflammatory response in predicting survival in patients with primary operable cancer. Future Oncol 6(1):149-163

13. Dolan RD, McSorley ST, Park JH et al (2018) The prognostic value of systemic inflammation in patients undergoing surgery for colon cancer: comparison of composite ratios and cumulative scores. Br J Cancer 119(1):40-51

14. Spence S, Gupta S, Alanie O, Ong J, Findlay H, Mahendra A (2019) Does the Modified Glasgow Prognostic Score Aid in the Management of Patients Undergoing Surgery for a Soft Tissue Sarcoma? World J Surg Surgical Res 2:1097

15. Dolan RD, Laird BJA, Horgan PG, McMillan DC (2018) The prognostic value of the systemic inflammatory response in randomised clinical trials in cancer: A systematic review. Crit Rev Oncol Hematol 132:130-137

16. Moyes LH, Leitch EF, McKee RF, Anderson JH, Horgan PG, McMillan DC (2009) Preoperative systemic inflammation predicts postoperative infectious complications in patients undergoing curative resection for colorectal cancer. Br J Cancer 100(8):1236-1239

17. Fang E, Wang X, Feng J, Zhao X (2020) The Prognostic Role of Glasgow Prognostic Score and C-reactive Protein to Albumin Ratio for Sarcoma: A System Review and Meta-Analysis. Dis Markers 2020:8736509

18. Hyldig N, Vinter CA, Kruse M et al (2019) Prophylactic incisional negative pressure wound therapy reduces the risk of surgical site infection after caesarean section in obese women: a pragmatic randomised clinical trial. BJOG 126(5):628-635

19. Keeney JA, Cook JL, Clawson SW, Aggarwal A, Stannard JP (2019) Incisional Negative Pressure Wound Therapy Devices Improve Short-Term Wound Complications, but Not Long-Term Infection Rate Following Hip and Knee Arthroplasty. J Arthroplasty $34(4): 723-728$

20. Newman JM, Siqueira MBP, Klika AK, Molloy RM, Barsoum WK, Higuera CA (2019) Use of Closed Incisional Negative Pressure Wound Therapy After Revision Total Hip and Knee Arthroplasty in Patients at High Risk for Infection: A Prospective. Randomized Clin Trial J Arthroplasty 34(3):554-559.e1

21. Bailey O, Shields DW, Donnelly I, Findlay H, Sharp E et al (2017) SUNstudy Study Protocol: Sarcoma Usual versus Negative pressure dressing Study Protocol. J Clin Exp Orthop 3(3):47

22. Sanniec KJ, Velazco CS, Bryant LA et al (2016) Immediate versus Delayed Sarcoma Reconstruction: Impact on Outcomes. Sarcoma 2016:7972318

23. Sanniec KJ, Swanson S, Casey WJ 3rd, Schwartz A, Bryant L, Rebecca AM (2013) Predictive factors of wound complications after sarcoma resection requiring plastic surgeon involvement. Ann Plast Surg 71(3):283-285
24. Merrill RD, Burke RM, Northrop-Clewes CA et al (2017) Factors associated with inflammation in preschool children and women of reproductive age: Biomarkers Reflecting Inflammation and Nutritional Determinants of Anemia (BRINDA) project. Am J Clin Nutr 106(Suppl 1):348S-358S

25. Raiten DJ, Sakr Ashour FA, Ross AC et al (2015) Inflammation and Nutritional Science for Programs/Policies and Interpretation of Research Evidence (INSPIRE). J Nutr 145(5):1039S-1108S

26. Martin L, Birdsell L, Macdonald N et al (2013) Cancer cachexia in the age of obesity: skeletal muscle depletion is a powerful prognostic factor, independent of body mass index. J Clin Oncol 31(12):1539-1547

27. Baracos VE, Arribas L (2018) Sarcopenic obesity: hidden muscle wasting and its impact for survival and complications of cancer therapy. Ann Oncol 29:1-9

28. Nakanishi R, Oki E, Sasaki S et al (2018) Sarcopenia is an independent predictor of complications after colorectal cancer surgery. Surg Today 48(2):151-157

29. Tamura T, Sakurai K, Nambara M et al (2019) Adverse Effects of Preoperative Sarcopenia on Postoperative Complications of Patients With Gastric Cancer. Anticancer Res 39(2):987-992

30. Dolan RD, Almasaudi AS, Dieu LB, Horgan PG, McSorley ST, McMillan DC (2019) The relationship between computed tomography-derived body composition, systemic inflammatory response, and survival in patients undergoing surgery for colorectal cancer. J Cachexia Sarcopenia Muscle 10(1):111-122

31. Kanda M, Kobayashi D, Tanaka C et al (2016) Adverse prognostic impact of perioperative allogeneic transfusion on patients with stage II/III gastric cancer. Gastric Cancer 19:255-263

32. Xiao H, Quan H, Pan S et al (2018) Impact of peri-operative blood transfusion on post-operative infections after radical gastrectomy for gastric cancer: a propensity score matching analysis focusing on the timing, amount of transfusion and role of leukocyte depletion. J Cancer Res Clin Oncol 144(6):1143-1154

33. Rimner A, Brennan MF, Zhang Z, Singer S, Alektiar KM (2009) Influence of compartmental involvement on the patterns of morbidity in soft tissue sarcoma of the thigh. Cancer 115(1):149-157

34. Garcia DR, Deckey D, Haglin JM et al (2019) Commonly Encountered Skin Biome-Derived Pathogens after Orthopedic Surgery. Surg Infect (Larchmt) 20(5):341-350

Publisher's Note Springer Nature remains neutral with regard to jurisdictional claims in published maps and institutional affiliations. 